\title{
The evolutionary significance of polyploidy
}

Yves Van de Peer ${ }^{1,2,3,4}$, Eshchar Mizrachi ${ }^{4,5}$, and Kathleen Marchal ${ }^{1,3,5,6}$

${ }^{1}$ Department of Plant Biotechnology and Bioinformatics, Ghent University, Technologiepark 927, B-9052 Ghent, Belgium.

${ }^{2}$ VIB-UGent Center for Plant Systems Biology, VIB, Technologiepark 927, B-9052 Ghent, Belgium

${ }^{3}$ Bioinformatics Institute Ghent, Ghent University, Technologiepark 927, B-9052 Ghent, Belgium

${ }^{4}$ Genomics Research Institute (GRI), University of Pretoria, Private Bag X20, Pretoria, 0028, South Africa

${ }^{5}$ Department of Genetics, Forestry and Agricultural Biotechnology Institute (FABI), University of Pretoria, Private Bag X20, Pretoria, 0028, South Africa

${ }^{6}$ Department of Information Technology, IDLab, imec, Ghent University, B-9052 Ghent, Belgium

Correspondence to Y. V.d. P. yves.vandepeer@psb.ugent.be

\begin{abstract}
Polyploidy, or the duplication of entire genomes, has been observed in both somatic and germ cells, and in both prokaryotic and eukaryotic organisms. Although the consequences of polyploidization are complex and variable, and seem to differ greatly between systems (clonal and non-clonal) and species, there is growing evidence that polyploidization correlates with environmental change or stress. Consequently, although often considered an evolutionary dead end, the short-term adaptive potential of polyploidization is increasingly being acknowledged. Furthermore, once established, the unique retention profile of duplicated genes following whole-genome duplication might explain important longer-term key evolutionary transitions and a general increase in biological complexity.
\end{abstract}

\section{Introduction}

Polyploid species (BOX 1) have been known for a long time. For instance, in plants, polyploidy has been studied for more than a hundred years, dating back to the early work of Hugo de Vries and G. Ledyard Stebbins, Jr. ${ }^{1}$. Nowadays, polyploidy is generally considered a common mode of speciation with farreaching consequences for plant evolution and ecology. Although polyploidy is much rarer in animals than plants, there are numerous cases of polyploid insects, fishes, amphibians and reptiles ${ }^{2,3}$. For a long time, ancient polyploidy events, dating back millions of years, were much less well documented than more recent events. Although, over 40 years ago, the Japanese geneticist Susumu Ohno ${ }^{4}$ proposed that two rounds of polyploidy occurred early in vertebrate evolution, it was not until the advent of genomics that conclusive evidence of ancient whole-genome duplication (WGD) events became available. The first clear indication for an ancient polyploidy - in brewers' yeast - was published less than 20 years ago 5 , followed shortly after by evidence that the plant model system Arabidopsis thaliana had also undergone a WGD during its evolutionary past ${ }^{6}$. At about the same time, two rounds of WGDs were proposed to have occurred during early vertebrate evolution ${ }^{7}$, as Ohno had predicted. In addition, a 
third WGD shared by all teleosts is thought to have occurred after fishes diverged from land vertebrates

${ }^{8}$. In recent years, many more remnants of ancient polyploidy events have been unveiled, largely owing to the sequencing of entire genomes and transcriptomes, and we now have evidence for the occurrence of tens, or even hundreds, of WGD events during the last 500 million years (My) of evolution (Fig. 1).

However, the number of species that are currently polyploid outnumbers the number of ancient WGDs by several orders of magnitude. This observation can partly be explained by the topology of a phylogenetic tree. Because most ancient lineages have become extinct, there are simply fewer places on the 'older' parts of a tree to observe a polyploidy event than there are on the tips of the tree. Furthermore, polyploid abundance is only expected to increase over time, since polyploidization is an irreversible process ${ }^{9,10}$. As a result, one would expect a higher incidence of polyploidy towards the tips of the tree than at the base. Still, because polyploidy is so prevalent, we would expect to find much more evidence of ancient WGDs ${ }^{11,12}$. The fact that there are many examples of recurrent polyploidy ${ }^{13}$ and many polyploids of fairly recent origin but relatively little evidence for ancient polyploidy events certainly within the same evolutionary lineage (Fig. 1) - provides an interesting paradox 2,11,14,15.

The relative paucity of polyploidy events that 'survive' and are established in the long run seems to suggest that polyploidy is usually an evolutionary 'dead end' $12,15,16$. However, at specific times in evolution, organisms that underwent and survived WGDs likely had some adaptive advantage, and outcompeted their diploid progenitors that did not undergo a WGD event, such that all descendants bear the trace of the WGD event, whereas all sister taxa died out. Therefore, it has been vividly discussed whether these WGDs, which in many cases characterize major lineages of organisms such as vertebrates, fishes, flowering plants, grasses and orchids, have survived by coincidence, and are randomly distributed over the evolutionary tree of life, or whether they may have occurred at very specific times, for instance during major ecological or environmental changes and/or periods of extinction ${ }^{17-19}$.

In this Review, we describe important WGDs that have occurred throughout the last $500 \mathrm{My}$ of evolution, and discuss the short-term survival and establishment of polyploids as well as their long(er)term evolutionary potential. We elaborate on neutral and adaptive processes that might associate WGD with survival and/or invasiveness, and which could be responsible for the increasingly observed pattern of WGDs and so-called decisive moments in evolution. Finally, we also briefly discuss the importance of polyploidy and WGD for non-germline and clonal systems such as prokaryotes and cancer. We do not discuss here how remnants of ancient duplication events can be unveiled, or the many different ways that gene duplicates can evolve novel gene functions but refer to many excellent reviews and papers on these topics for further information ${ }^{20-23}$.

\section{Short term effects of WGDs}

It has long been known that polyploidy can have detrimental effects. Indeed, 'genomic shock', caused by genomic instability, mitotic and meiotic abnormalities, and gene expression and epigenetic changes following WGD can lead to increased sterility and decreased fitness. The harmful effects of WGD have been extensively discussed and reviewed before ${ }^{24}$. Here, we want to focus on the adaptive potential of polyploids (Fig. 2). The reasons why new polyploids might be able to survive and even thrive have also long been recognized ${ }^{24-29}$ and will therefore only briefly be reiterated here. First, in order to survive, one major hurdle needs to be overcome, namely minority cytotype disadvantage. Newly evolved polyploids need to find polyploid partners to mate with in an environment where they are usually surrounded by diploids. However, at least in plants, polyploidization is often accompanied by a transition from cross-pollination to self-pollination or from sexual to asexual reproduction, which helps 
to overcome the minority cytotype disadvantage and, therefore, assortative mating by cytotype could enhance the short-term survival of polyploids ${ }^{2}$ (see also BOX2).

\section{The adaptive potential of 'new' polyploids}

Second, most explanations for the short-term success of polyploids centre around the effects of genomic changes and increase in genetic variation, brought about by changes in gene expression and epigenetic remodelling following polyploidization ${ }^{30-37}$. Increased genetic variation can potentially affect the morphology, physiology, and ecology of newly formed polyploids 26,33,38. Such changes in morphology and physiology could in turn impact interspecies interactions. For instance, divergence in traits attractive to pollinators can lead to differentiation in pollinator communities ${ }^{38}$, causing isolation of diploids and polyploids even within mixed populations, and facilitate polyploid establishment ${ }^{39}$. It is known that the colour and scent of flowers can differ in polyploids versus diploids ${ }^{40,41}$. It is reasonable to assume that the preference of certain pollinators for polyploids based on these traits can help overcome minority cytotype exclusion and contribute to the reproductive isolation of polyploid lineages and thus to their speciation and establishment. Interactions with herbivores may also change after polyploidy. It was recently shown that neofunctionalization of genes following WGD events in the Brassicales probably allowed plants to escape herbivory ${ }^{42,43}$. Although neofunctionalization of genes does need some time to evolve, one could easily imagine short term effects of polyploidy on herbivory as well, due to immediate changes in secondary metabolism ${ }^{30,44}$. Immediate changes following transitions to polyploidy were for instance recently demonstrated by comparing the metabolic profiles of natural diploids and artificial colchicine-induced autotetraploids of Arabidopsis thaliana. Such comparison showed important differences between diploids and polyploids in the concentration of metabolites related to the tricarboxylic acid cycle (TCA) and gamma-amino butyric acid (GABA) shunt ${ }^{44}$. In addition, genes related to the TCA and GABA shunt were differentially expressed between diploids and autotetraploids. The functional roles of TCA and GABA metabolites are diverse but differences in concentrations could be expected to have important adaptive consequences for the specific ecology of diploids and polyploids. Changes in metabolite profiles and possible consequent biotic interactions might also explain why polyploids show increased resistance to pathogens ${ }^{45}$. It has long been known that polyploid crops and ornamental plants can show increased resistance to fungal pathogens, nematodes, and insects, although sometimes also the opposite has been observed $\left({ }^{26}\right.$ and references therein).

The effects of increased genetic variation can also increase the tolerance for a broader range of ecological and environmental conditions, although this remains a topic of some controversy ${ }^{2,17,26,46}$. For instance, it has repeatedly been proposed that polyploids have higher stress tolerance (environmental robustness), which finds support in the observation that present-day polyploids often seem to occur at higher frequencies in new, disturbed or harsh environments ${ }^{27,47,48}$. An example is given in Fig. 3, showing the different distribution of diploid and tetraploid Neobatrachus (frog) species in Australia. However, most of the 'evidence' that WGDs facilitate or accelerate adaptation, for instance to new or changing environments, is circumstantial (see further) and direct tests are difficult to conduct and thus are rare, although there have been some studies exploiting the effect of polyploidy on biotic and abiotic stress. For instance, Chao et al. ${ }^{49}$ found that, in naturally occurring tetraploid Arabidopsis thaliana accessions, polyploid plants appear to confer increased salt tolerance by regulating leaf potassium levels. Other tetraploid plants such as rice and citrus have also been shown to have an increased tolerance to salt and drought stress, due to polyploidy affecting the expression of genes involved in stress and hormone response pathways ${ }^{50,51}$. 


\section{WGD and species diversification}

Polyploidization seems, at least in plants, to occur at relatively high frequency and the latest estimates suggest that up to 25 - 30\% of extant flowering plants are 'young' polyploids ${ }^{10,52,53}$. On the other hand, recent simulations on the formation of polyploids have shown that the likelihood of new allopolyploids - and most likely also autopolyploids (see BOX 1) - getting established at their site of origin is very low, and depends on many different factors, such as niche separation from their diploid progenitors, population size, fecundity and self-fertilization ${ }^{54}$. This seems to support earlier work on plants suggesting that polyploids form new species more slowly and go extinct more quickly, and consequently have lower diversification rates than their diploid relatives ${ }^{16,55}$. This is probably even more so in stable environments, where most of the diploid progenitors are well-adapted and polyploids have a hard time competing ${ }^{56}$. In addition, although polyploidy is generally believed to be an important mechanism of sympatric speciation, at least in plants ${ }^{3}$, conditions for species formation and diversification through WGD are likely not always instantaneous and selection to strengthen prezygotic barriers in new polyploids is probably critical for the establishment of polyploid species in sympatry ${ }^{57}$. Schranz et al. ${ }^{58}$ presented a model (the WGD radiation lag-time model) that, based on general knowledge of numbers of species in certain plant clades, hypothesizes that WGDs do often result in higher diversification rates, but after a delay of potentially up to several millions of years. Recently, Tank et al. ${ }^{59}$ investigated the link between potentially increased rates of diversification and well-documented ancient WGDs, and found significant statistical support for a non-random association between WGD events and a delayed increase in rates of diversification. Other studies, focusing on the WGD in the teleosts and considering the diversity of teleosts and holosteans (gars and bowfin) in the fossil record, failed to detect an association between genome duplication and significant shifts in rates of lineage diversification, even considering longer time frames ${ }^{60}$. A recently proposed model, LORe (Lineage-specific Ohnolog Resolution') ${ }^{61}$, suggests that functional divergence of genes, assumed to be responsible for specific adaptations and diversification, can be delayed for tens of millions of years, due to delayed rediploidization, which is particularly relevant for autopolyploidy ${ }^{62}$. Under LORe, the rediploidization process is not completed until after a speciation event, which will result in the independent divergence of 'ohnologs' (paralogs retained from WGD) in sister lineages. Delayed species radiation post-WGD is then explained by functional divergence of genes under or coinciding with unique environmental or ecological conditions. As such, LORe, as a product of highly delayed rediploidization, offers a mechanism to explain time-lags between WGD events and subsequent lineage-specific diversification ${ }^{61}$.

All in all, it is probably safe to say that the discussion about the relationship between polyploidy and speciation and species diversification has not been settled yet, mainly because the causative link between WGDs and rates of species diversification is still unclear ${ }^{16,63-65}$.

\section{WGDs and decisive moments in evolution}

As described above, polyploids can overcome the cytotype minority disadvantage, sympatrically speciate, and have at least the potential for quick adaptation due to their often more plastic genomic background ${ }^{26,37}$. All this could explain the short-term survival of new polyploids and the fact that recent polyploids abound. On the other hand, ancient WGDs surviving for at least a few tens of millions of years seem much rarer. There is only a handful for animals and fungi, while the number of known ancient WGDs in plants is considerably larger, but, interestingly, many of the ancient WGDs are found in separate lineages, while the number of recurrent polyploidy events - thus within the same evolutionary 
lineage - is usually limited (Fig. 1). The question then arises as to whether the observed paleopolyploidies survived by pure coincidence and are randomly scattered over the tree of life, or whether they occurred at very specific times in evolution, such as during times of environmental upheaval or periods of extinction.

\section{WGDs and mass extinctions}

The increased mutational and environmental robustness and increased potential for specific adaptation of polyploids, as described above, might reduce the risk of extinction during periods of environmental turmoil $^{17,66}$. The most compelling evidence that WGD might play a role in avoiding extinction probably comes from flowering plants. Recent analyses of a large number of genome and transcriptome sequence data suggest a wave of WGDs that seems to have occurred close to the Cretaceous-Paleogene ( $\mathrm{K} / \mathrm{Pg}$ ) boundary ${ }^{15,18,64,67-69}$ (Fig. 1). The K/Pg boundary is marked by a number of cataclysmic events such as a meteor impact near Chicxulub (Mexico) and a possibly impact-induced increase in Deccan flood volcanism (India) ${ }^{70}$. These cataclysmic events caused major climate change and global warming ${ }^{71}$ leading to the extinction of 60 to $70 \%$ of all plant and animal life, including all non-avian dinosaurs. Interestingly, the environmental instability during the K/Pg boundary might have also fostered polyploid speciation in the amphibian clade that includes Hylids, Ceratophrids, Cycloramphids, Leptodactlids, and Bufonids, all of which contain multiple independent diploid-polyploid species pairs, and diverged about 65 mya $^{2}$.

A potentially broader ecological tolerance (see above) seems to fit well with a clustering of paleopolyploids around the K/Pg boundary. Other polyploidy events mapped on the phylogenetic tree shown in Fig. 1 might correlate with extinction events or periods of environmental change as well, but are much harder to prove. In order to be able to observe a potential correlation, and find statistical support, many data points are needed ${ }^{68}$. For instance, although polyploidy in gymnosperms is rare and there are no indications for WGDs in gymnosperms at the K/Pg boundary, with the possible exception of Welwitchia ${ }^{72}$, it has recently been suggested that some older WGDs observed in gymnosperms might overlap with the Permian-Triassic extinction ${ }^{72}$. Although it is indeed again tempting to speculate that polyploid conifers might have had a better chance of survival and outcompeted their diploid sister species at a time that many of these conifer clades originated, these questions might actually never be fully resolved, since there are few (remaining) branches at the Permian-Triassic boundary and therefore few data points to date (see Fig. 1).

The same holds true for the two rounds of WGDs during early vertebrate evolution, 500 - 550 Mya, which could be close to the Cambrian explosion ${ }^{73}$, and the subsequent WGD event in teleosts, which may have occurred close to the Permian - Triassic mass extinction event, $250 \mathrm{Mya}^{74}$. Accurate dating of such ancient events that have possibly occurred several hundreds of millions of years ago is difficult and prone to errors ${ }^{14,68}$. Furthermore, to quote Soltis and Burleigh ${ }^{75}$, "we must be careful not to be carried away by the excitement of discovering more and more ancient WGDs and being tempted to look for correlations". On the other hand, if we believe the correlation between many of the WGDs in plants and the K/Pg extinction to be real, the majority of the WGDs in plants seem to represent a quite unique event, which makes 'coincidental' WGDs and their establishment even harder to explain. Also for animals, it has been previously suggested that polyploidization is correlated with periods of climate change and unstable environments. For instance, all animal taxa in which 'recent' polyploidy events have been described are ectothermic (do not regulate their body temperature) and are therefore susceptible to the changes in environment. A striking feature shared by polyploid fish and amphibians 
is external reproduction in freshwater environments, mainly in regions where temperature fluctuations during the breeding season are common ${ }^{2}$.

\section{More 'waves' of WGDs}

Another example of a more recent wave of WGDs that might be correlated with a period of change and that is supported by a large number of data points has recently been described by Estep et al. ${ }^{76}$, and concerns the repeated formation of polyploids correlating with the expansion of $\mathrm{C}_{4}$ grasslands in the Late Miocene. Grasses using $\mathrm{C}_{4}$ photosynthesis became important and largely displaced $\mathrm{C}_{3}$ grasslands in the Late Miocene, after an earlier decrease of atmospheric $\mathrm{CO}_{2}$ levels in the Oligocene (23 to $33.9 \mathrm{Mya}$ ) and a forest-to- $\mathrm{C}_{3}$-grassland transition in the Early-Middle Miocene (5.3 to $\left.23 \mathrm{Mya}\right)^{77}$. In the grass tribe Andropogoneae, the authors found more than $30 \%$ of the $\sim 1,200$ species to be allopolyploids, the result of a minimum of 34 distinct polyploidy events, most of which occurred during the expansion of the $\mathrm{C}_{4}$ grasslands. Polyploidy hence also seems to be correlated with dominance of $\mathrm{C}_{4}$ over $\mathrm{C}_{3}$ grasses and largescale displacement of the latter ${ }^{76}$. Interestingly, WGDs in the Early-Middle Miocene have recently also been reported for several tribes of the Asteraceae ${ }^{64}$. Again, global grassland expansion and a changing environment might have created novel open niches for polyploids to invade (see further).

Besides the already previously documented paleopolyploidy events in the crucifer lineage (including e.g. Arabidopsis, Fig. 1), transcriptome data obtained by next generation sequencing recently showed that several species in the Brassicaceae lineage have undergone a further more recent ( 7 to 12 Mya) polyploidy event. Kagale et al. ${ }^{19}$ identified eight WGDs corresponding to at least five independent polyploidy events. Although the Brassicaceae family diverged from other eudicots at the beginning of the Cenozoic era (60 Mya), major diversification occurred only during the Neogene period (0 to $23 \mathrm{Mya}$ ). Again, both species divergence and the occurrence of several WGDs seem to be clustered in time around periods that were characterized by prolonged unstable climatic conditions. Such a correlation between WGD events and diversification of many Brassicaceae species again provides support for the importance of environmental change in the establishment of polyploidization. Finally, many of the extant polyploids that we are familiar with today might be relics of the latest glaciations. Indeed, (allo)polyploids are often found in deglaciated habitats (see further, polyploidy and shifts in ecological niches) as a result of hybridization (secondary contact) of closely related species that had earlier become separated as a result of glacial barriers ${ }^{78,79}$.

Apart from an increased adaptive potential, more neutral processes might also have an important contribution to polyploidy at times of environmental upheaval or extinction. Indeed, it is welldocumented that the production of unreduced gametes can be increased by external stimuli such as stress and a fluctuating environment. In particular temperature has a pronounced effect on unreduced gamete formation (see BOX1 and references therein). It is therefore likely that both adaptive and neutral processes contributed to the establishment of polyploids at times of environmental change (Fig. 4).

\section{Polyploidy and (shifts in) ecological niches}

A higher tolerance for a broader range of ecological and environmental conditions, as often attributed to polyploids due to novel and varying phenotypes that may predispose polyploid lineages to colonization success in novel environments (e.g. Fig. 3), as well as subsequent adaptation due to increased genetic variation while restoring sexual reproduction through selfing or apomixis, could certainly have been advantageous for exploiting new and changed ecosystems ${ }^{26,80,81}$. For instance, 
Pandit et al. ${ }^{82}$ found that polyploids are $20 \%$ more likely to be invasive than closely related diploids when they compared ploidy levels among rare and invasive plant species on a worldwide scale. We have argued before that the availability of new ecological niches could be the single most important determinant for the survival and long-term evolutionary success of a WGD ${ }^{17}$. The potential for increased phenotypic and genotypic diversity provided by WGDs is less useful when there are no niches in which the new phenotypes or genotypes are advantageous. In stable ecosystems, newly formed polyploids are probably not able to compete with the highly adapted occupants of existing niches, including their diploid ancestors ${ }^{56}$.

On the other hand, to what extent polyploids establish through niche differentiation and polyploidy promotes ecological shifts needs further investigation ${ }^{83}$. For instance, in a study testing for climate shifts between diploids and polyploids, rather than a clear pattern of niche shifts, niche conservation and contraction was observed. Glennon et al. ${ }^{84}$ compiled a data set of 20 different plant species distributed in North America and Europe that harbour diploid and young polyploid species, and used statistical tests of niche overlap to compare climate conditions of diploids and polyploids of each of the 20 species. Since the results of their test did not support frequent or strong climate-based niche shifts by polyploids, they assumed other processes (such as short or long range dispersal mechanisms) to be more important to explain polyploid establishment and survival than climate-related factors. In another carefully executed study, Marchant et al. ${ }^{85}$ used a variety of niche analysis and multivariate techniques to investigate the geographic distribution, niche breadth and niche overlap, to infer patterns of niche distribution between allopolyploids (10 ferns and three angiosperms) and their diploid progenitors. The authors hypothesized that the allopolyploids would occupy broader (because of increased allelic diversity) and distinct ecological niches relative to those of their progenitors. However, quite unexpectedly, all allopolyploids, with one exception, had a high overlap with at least one of its progenitors. Only one polyploid clearly showed niche novelty ${ }^{85}$. Interestingly, another study of four related European primrose species of different ploidy levels (diploid, tetraploid, hexaploid and octoploid), using seven bioclimatic variables to quantify niche overlap between species, and using a series of univariate and multivariate analyses combined with modelling techniques, found that polyploids, as expected, did occupy ecological niches that differed from those of their diploid relatives

79. However, contrary to expectations, the authors also observed that the polyploids of one species occupied narrower environmental and geographical spaces than their diploid relative, which goes against the idea that polyploids generally have broader niche occupancies.

WGDs that have occurred early in the evolution of the flowering plants ${ }^{86}$, or prior to the divergence of the eudicots ${ }^{87}$ or monocots ${ }^{88}$, do not, as far as we can tell, seem to coincide with periods of mass extinction, and it is questionable whether they rose to ecological dominance by filling niches that became available after a drastic change of the environment. A more credible explanation here is that polyploids and their descendants filled niches that might already have existed but that remained largely unoccupied in the past because the necessary phenotypic traits for survival in that niche had not yet been developed. WGDs might have led to so-called key innovations (see further, long-term effects of WGDs) ${ }^{86,89}$, facilitating diversification and radiations and the invasion of previously unoccupied niches at later times.

\section{Long term effects of WGDs}

\section{Duplicability and retention of genes following WGD}


The question remains whether polyploidy or WGD has specific long-term effects for evolution, in addition to some selective advantage explaining the short-term survival and establishment of polyploids, as discussed above. Duplicate genes that originated in the two successive ancient WGD events (around 500 - 550 MYA) in vertebrates (Fig. 1) have been shown to still make up around $30 \%$ of the genes in the human genome ${ }^{90}$. These genes are thought to be particularly sensitive to dosagebalance, unlikely to vary in copy number, and preferentially associated with disease ${ }^{91}$. Subsequent studies suggested that these duplicate genes are likely heavily susceptible to dominant deleterious mutations ${ }^{92}$. Functionally, these genes are involved in organismal complexity, and play important roles in signalling and development, as well as transcriptional regulation. Recently, Singh et al. ${ }^{93}$ performed a comparative analysis of six vertebrate species and six outgroup genomes, and identified around 2000 ohnolog gene families. These ohnologs were extremely conserved in terms of copy number, with virtually all remaining as four copies in the six genomes ${ }^{93}$. Ohnologs were approximately three-fold more likely than non-ohnologs to account for cancers and autosomal dominant diseases, and up to 8fold more likely to be enriched in genes with autoinhibitory protein folds. So it seems that, at least in vertebrates, genes involved in form and development are often under purifying selection, and are 'dangerous duplicates' 92 that must be retained. In support of this, following the rainbow trout WGD (dated to around $80 \mathrm{Mya}$ ), genes showed a return to singleton status in at least $50 \%$ of the cases, but others mainly retained duplicate copies for the same so-called 'dangerous genes' ${ }^{44}$. A recent study on the tetraploid frog Xenopus laevis (an allopolyploid that is functionally diploid due to lack of recombination between homeologs) once again demonstrated that genes retained in multiple copies after the ancient vertebrate and teleost genome duplications were also more likely to be retained as ohnologs in X. laevis ${ }^{95}$.

From vertebrates we learn that some WGD-derived duplicate genes must obligatorily be retained as duplicates. But what happens when you roll the dice multiple times? Considering many independent ancient WGDs, are the same genes repeatedly maintained as duplicates over long periods of evolutionary time? In addition to the curiously high number of independent WGDs observed around the K-Pg boundary, flowering plants offer perhaps a unique system in all of the known Eukaryotic tree of life in which to observe the survivability of duplicated genes following many independent WGD events. Li et al. ${ }^{96}$ recently investigated the fate of duplicated genes for almost 40 different flowering plant species, all of which have experienced one or more ancient WGDs. It was observed that gene loss following WGD strongly deviated from a stochastic model, with a majority of genes to rapidly return to single-copy status ${ }^{96}$. On the other hand, some genes were found to be consistently present as multi-copy. In general, these represent gene families that are involved in conditional responses to biotic and abiotic stress and are important for local adaptation. Duplicated genes belonging to these gene families are quickly put to novel use, whether created by both WGD or small-scale duplication ${ }^{97}$. Another group of genes that often show biased retention following WGDs again represent genes encoding proteins that act in multiprotein complexes and/or play important roles in signalling, development, and transcriptional regulation ${ }^{96}$. Aside from the dominant-negative hypothesis of retention, as mentioned above, this pattern of gene loss and retention can be explained by dosage-balance constraints and selection against loss of individual components of completely duplicated macromolecular complexes and/or pathways, because this would disrupt their overall stoichiometry ${ }^{97-100}$.

\section{Biased retention of genes and long-term consequences}

The strongly biased retention of regulatory and developmental genes subsequent to WGD is bound to have important consequences for evolution on the longer term. Initially, the retention of these 
particular genes may be considered an evolutionary spandrel - a neutral by-product ${ }^{98}$ (Fig. 4). However, eventually, selection to maintain dosage balance and stoichiometry will relax over time allowing duplicated networks to be rewired and to evolve novel functionality and increase biological complexity 101,102. Indeed, recent work considered the potential impact of WGDs on the evolution of transcription factors (TFs) in metazoans and showed that their longer retention time following WGDs was necessary for TFs to gain novel functions ${ }^{103}$. Therefore, we can consider the long-term or delayed impact of WGDs on innovation and diversification as the product of non-adaptive negative selection (leading to biased retention of classes of genes such as transcriptional regulators), neutral processes (such as for instance domain rearrangements), and subsequent positive selection (such as neo- or subfunctionalization) on genes and networks. In conclusion, as long as there is sufficient time for the polyploid to survive a WGD, there is indeed a plausible mechanism where neutral disentanglement of dosage-sensitive components and freedom to explore complexity can result in adaptive diversity, leading to specialization and possibly speciation (Fig. 4). But widespread speciation after WGD would therefore typically be a delayed phenomenon ${ }^{17}$. This would also be consistent with the 'WGD-radiation lag time model' first proposed by Schranz et al. ${ }^{58}$.

\section{Polyploidy in clonal systems}

Sometimes, polyploidy does not affect whole organisms, but arises in somatic cells of otherwise diploid organisms, where it can play 'scheduled' roles in development and stress responses ${ }^{37}$. Somatic polyploid cells can arise either by cell fusion or when cell division aborts before cytokinesis ${ }^{104}$. Such cells are known as endopolyploid. Often, endopolyploid cells arise in diploids as a normal part of development. Well known examples of specialized endopolyploid cells are for instance fibres and leaf hairs in plants ${ }^{105}$, or megakaryocytes, glial cells, and wing scale cells in moths and butterflies ${ }^{106,107}$. Also during liver growth, the frequency of tetraploid and octoploid hepatocytes steadily increases. Hepatocytes are particularly prone to polyploidization in response to DNA damage, where most other cells would undergo apoptosis ${ }^{108}$. Although the benefits of programmed polyploidy are not fully understood, the ability to generate endopolyploid cells is likely an important adaptation when rapid growth or large cell size are required, or in tissues or cell types where mitotic division would be deleterious for structural reasons ${ }^{108}$. Endopolyploidy can also be induced by variable conditions, such as drought or salt stress. In this respect it is interesting to note that somatic WGD might contribute to stress resilience in plants, which might even translate to the whole-organism effects described above (see short term effects of WGDs). For instance, in sorghum it has been demonstrated that root endopolyploidy is associated with salt tolerance ${ }^{109}$, suggesting that the ability to induce endopolyploidy may be directly responsible for the resistance to salt, likely due to cell size changes in the roots. The greater salt tolerance of Arabidopsis autopolypoloids ${ }^{49}$ might, at least in part, be explained by similar processes ${ }^{110}$.

\section{Polyploidy and clonal (eukaryotic) systems}

Unscheduled - and thus not part of normal development - WGDs occur as well and for instance characterize a substantial fraction of human tumours. Aneuploid cancers displaying triploid or near tetraploid genomes likely originate from a transient tetraploid intermediate ${ }^{111}$. Such transient polyploidy states have been observed in several tumour types at early stages of tumorigenesis ${ }^{111-113}$. The origin of tetraploidy in tumours has been attributed to cell fusion events, errors in the progression or exit from mitosis, or as a consequence of persistent damage response or telomere dysfunction ${ }^{108}$. 
Tetraploidization has been associated with several mutational events, for instance in the tumour suppressor APC, in which mutations lead to colon cancer ${ }^{114,115}$, and defects in p53 and Rb tumour suppressor pathways ${ }^{108,116}$.

Transient tetraploids are unstable (CIN, chromosomal instability), and frequent chromosomal missegregation results in the acquisition of aneuploidy over short periods ${ }^{108,117}$. In addition, the polyploid state of a cell has been associated with a higher propensity for developing chromotripsis, where, in contrast to the gradual evolution of aneuploidy, massive DNA structural rearrangements occur in localized chromosomal regions as the result of a single catastrophic event ${ }^{118}$. Because of their genomic instability, transient tetraploids/aneuploids proliferate better in cells with damaged apoptosis or cell cycle checkpoint pathways. This explains why somatic events such as Rb and TP53 alterations often precede ${ }^{119}$ or are associated with a WGD event ${ }^{108,120}$ (Fig. 4).

Tetraploids and their subsequent aneuploidy state rapidly give rise to adaptive phenotypes resulting in tumorigenesis ${ }^{108,112,113}$ or drug resistance (111 and references therein). Also in 'clonal' yeasts such as Cryptococcus neoformans and Candida albicans, aneuploidies that result in adaptive drug resistance have been generated through a transient polyploid state ${ }^{121}$. These recent findings show that in clonal systems the transient tetraploid state is a 'gateway karyotype' that, as in plants, increases genetic variation and adaptive potential, but in contrast to plants does so mainly by promoting aneuploidy ${ }^{108,111}$. Indeed, aneuploidy and chromosomal duplications have been associated with high adaptive potential in clonal systems ${ }^{111,122,123}$. Experimental evolution studies in yeast showed for instance that specific chromosome duplications can confer a fitness advantage under stressful conditions ${ }^{122,124-126}$. Changes in copy number result in an altered expression that can give rise to an adaptive phenotype if the proper genes are amplified ${ }^{111,125}$. Also in cancers, aneuploidy, mainly as a consequence of CIN, would contribute to tumorigenesis and progression, for instance by promoting the loss of tumour suppressors, the amplification of oncogenes, or the formation of fusion genes ${ }^{120,127,128}$ (Fig. 4).

However, WGD does not only increase adaptive potential by providing a gateway karyotype that gives rise to CIN and increased genetic variation. About $90 \%$ of the solid tumours and $50 \%$ of the hematopoietic cancers are aneuploid and display $\mathrm{CIN}{ }^{128}$ and according to a pan-cancer analysis only about $37 \%$ would have undergone a WGD ${ }^{127}$. Nevertheless, near tetraploid cells have been shown to have a higher tumorigenic capacity ${ }^{129}$. Genome doubling in cancer is often associated with poor prognosis and disease relapse ( ${ }^{129}$ and refs therein). In addition, several studies have shown that resistance to targeted therapy in tumour cells is associated with the origin of polyploidy ${ }^{111}$. Also, in yeast it has been shown that tetraploids adapt faster than haploids or diploids ${ }^{125}$. Part of this higher evolvability in tetraploid clonal cells can be attributed to the fact that the higher polyploidy itself increases robustness against further genetic alterations as more genome copies will compensate for gene deletions or inactivating genetic variations ${ }^{108,111}$. Although $\mathrm{CIN}$ and aneuploidies can drive tumorigenesis or adaptive phenotypes, they are often more likely to give rise to proteotoxicity and/or generate a deleterious phenotype ${ }^{128}$. However, the polyploid state resulting from a tetraploid intermediate is believed to buffer the effects of aneuploidies. Dosage effects of an aneuploidy are expected to be relatively less severe in a $4 n$ than in a $2 n$ background and many of the extreme phenotypes resulting from aneuploidy are attenuated in polyploid cells ${ }^{123}$. As a result, tetraploids are expected to generate a higher genetic variability by providing higher tolerance for $\mathrm{CIN}$, as was indeed shown by Dewurst et al. ${ }^{129}$ for colorectal cancer. This higher tolerance might also explain why chromothripsis could be associated with hyperploid but not with diploid cells ${ }^{118}$. Consequently, some of the adaptive genetic variation is expected to depend on the polyploid background, which provides the proper buffering. This ploidy-dependent beneficial effect of aneuploidies was indeed confirmed by 
Selmecki et al. ${ }^{125}$, who showed that specific adaptive aneuploidies only conferred a fitness effect in a $4 n$ but not a $2 n$ background.

Lastly, because polyploidy buffers the effects of partially recessive deleterious mutations, such mutations can accumulate and create standing variation that might be adaptive in a new environment (drug resistance ${ }^{117}$ ) or that allows cells to explore adaptive solutions involving epistatic interactions ${ }^{130}$. As such, transient tetraploidy can, by generating and buffering aneuploidy, result in a better long-term adaptation (Fig. 4). Interestingly, Selmecki et al.'s ${ }^{125}$ study showed that $4 \mathrm{n}$-evolved clones that became near diploid had a higher fitness than $2 \mathrm{n}$-evolved clones. Temporal studies in lung cancer showed that a WGD preceded large-scale subclonal diversification, indicating that a genome doubling also accelerates adaptation at later stages of cancer evolution ${ }^{119}$ (Fig. 4).

\section{Polyploidy in prokaryotes}

Recent studies show that also in Bacteria and Archaea, in contrast to what has generally been assumed, polyploidy is not all that uncommon ${ }^{131-133}$. Polyploidy seems to have evolved independently several times during evolution in different groups of Archaea and Bacteria for different evolutionary reasons ${ }^{132,134}$. As polyploidy has mostly been studied in the context of Archaea and Bacteria that live in extreme conditions ${ }^{134}$, some of the proposed benefits of polyploidy might be confined to these extremophiles, such as providing resistance against severe conditions that introduce double-strand DNA breaks (X-ray radiation, desiccation), or providing a phosphate storage that would allow cells to divide a few times in the absence of external phosphate (Fig. 4). However, proposed benefits that relate to gene dosage and mutational robustness might be more widespread in the bacterial kingdom, although so far, little experimental evidence is available ${ }^{134}$. Just like in higher eukaryotes, polyploidy in prokaryotes would result in gene redundancy and subsequent mutational robustness, which would allow changing some of the gene copies without losing the wild-type information of the remaining ones. The standing variation thus generated might allow cells to grow under unfavourable conditions that inhibit growth of the homozygous wild-type. Gene conversion or the non-reciprocal transfer of information between homologous sequences through homologous recombination would play an important role in establishing the adaptive benefit of polyploidy-dependent mutational robustness in prokaryotes ${ }^{135}$ and maybe (some) eukaryotes as well ${ }^{136}$ - because it would equalize genome copies to yield a genome that is homozygous for functional genes, thereby selecting the favourable alleles under selective conditions and removing unwanted ones, even under non-selective conditions to prevent the accumulation of deleterious alleles that, on the long term, would drive the clonal population extinct. Gene conversion is of particular importance in those polyploid organisms that more or less randomly distribute chromosomes over daughter cells, because such a random assortment does not guarantee that compensatory alleles located on different chromosomes will be co-inherited in a subsequent generation. Random assortment thus often results in unviable offspring and in the lack of evolutionary constraints to select for combinations of beneficial alleles located on different chromosomes. The evolution of more accurate chromosome distribution during cell division (i.e. emergence of mitosis), such as recently suggested by Markov and Kaznacheev ${ }^{137}$ could provide an efficient mechanism to protect ancestral amitotic polyploids against the accumulation of segregation load.

\section{Conclusions}

The possibility of sequencing entire genomes and transcriptomes at low cost, together with the potentially large impact of WGDs on speciation and biodiversity dynamics, ecology, and evolution in 
general, has created a recent tsunami of studies and papers, including some entire journal issues (or large parts thereof) devoted to polyploidy ${ }^{138-140}$. One of the main conclusions in reviewing the vast literature on polyploidy and WGDs is that the 'consequences of polyploidy' are complex and variable, and seem to differ greatly between different species ${ }^{141-143}$. Also reconciling the short term effects of polyploidy with subsequent long-term evolution and successful establishment of polyploids needs much more research still ${ }^{11,75,85,139}$. On the other hand, there seems to be accumulating evidence linking shortterm polyploid establishment and survival to periods of environmental and ecological upheaval. If true, this of course has the potential to help explain the apparent contradictory evolutionary fates of polyploids: polyploidy is usually an evolutionary dead end, except under 'abnormal' circumstances when polyploids might have an edge over non-polyploids. Furthermore, once established, due to the biased retention of duplicated genes, polyploidy might also have important consequences for long-term evolution. Since specifically regulatory and developmental genes are being retained, linking WGD with important evolutionary transitions and a general increase in biological complexity, as already proposed more than 40 years ago by Susumu Ohno ${ }^{4}$, might not be so far-fetched.

Also cancer is more and more seen as an evolutionary system with a role increasingly being ascribed to polyploidy. Indeed, in cancer, but also in other eukaryotic clonal 'systems' such as yeast, polyploidy plays a crucial role in increasing the adaptive potential during periods of extreme stress through providing mutational robustness and increasing the adaptive effects of CIN. Even in prokaryotes, which, for a long time were believed to be strictly monoploid, polyploidy is prevalent and assumed to mediate gene dosage regulation and/or to provide cells with sufficient standing variation to quickly adapt under changing conditions or environments.

The impact of WGD on evolutionary events and adaptations might thus be much more widespread than initially thought. Consequently, a better understanding of polyploidy and WGD will undoubtedly be important to tackle future challenges in areas as diverse as global warming and climate change, agriculture and crop domestication (most of our crops are polyploids), natural selection and adaptation, and cancer research. 


\section{References}

1 Soltis, D. E., Visger, C. J. \& Soltis, P. S. The polyploidy revolution then...and now: Stebbins revisited. Am J Bot 101, 1057-1078, doi:10.3732/ajb.1400178 (2014).

2 Mable, B. K., Alexandrou, M. A. \& Taylor, M. I. Genome duplication in amphibians and fish: an extended synthesis. J Zool 284, 151-182 (2011).

Otto, S. P. \& Whitton, J. Polyploid incidence and evolution. Annu Rev Genet 34, 401-437, doi:10.1146/annurev.genet.34.1.401 (2000).

4 Ohno, S. Evolution by gene duplication. (Springer Verlag, 1970).

5 Wolfe, K. H. \& Shields, D. C. Molecular evidence for an ancient duplication of the entire yeast genome. Nature 387, 708-713, doi:10.1038/42711 (1997).

Arabidopsis Genome, I. Analysis of the genome sequence of the flowering plant Arabidopsis thaliana. Nature 408, 796-815, doi:10.1038/35048692 (2000).

Dehal, P. \& Boore, J. L. Two rounds of whole genome duplication in the ancestral vertebrate. PLoS Biol 3, e314, doi:10.1371/journal.pbio.0030314 (2005).

Amores, A. et al. Zebrafish hox clusters and vertebrate genome evolution. Science 282, 1711-1714 (1998).

Meyers, L. A. \& Levin, D. A. On the abundance of polyploids in flowering plants. Evolution 60, 1198-1206 (2006).

Scarpino, S. V., Levin, D. A. \& Meyers, L. A. Polyploid formation shapes flowering plant diversity. Am Nat 184, 456-465, doi:10.1086/677752 (2014).

Soltis, P. S., Marchant, D. B., Van de Peer, Y. \& Soltis, D. E. Polyploidy and genome evolution in plants. Curr Opin Genet Dev 35, 119-125, doi:10.1016/j.gde.2015.11.003 (2015).

Arrigo, N. \& Barker, M. S. Rarely successful polyploids and their legacy in plant genomes. Curr Opin Plant Biol 15, 140-146, doi:10.1016/j.pbi.2012.03.010 (2012).

Soltis, D. E. \& Soltis, P. S. Polyploidy: recurrent formation and genome evolution. Trends Ecol Evol 14, 348-352 (1999).

Campbell, M. A., Ganley, A. R. D., Gabaldón, T. \& Cox, M. P. The case of the missing ancient fungal polyploids. American Naturalist, doi:10.1086/688763 (2016).

Vanneste, K., Maere, S. \& Van de Peer, Y. Tangled up in two: a burst of genome duplications at the end of the Cretaceous and the consequences for plant evolution. Philos Trans R Soc Lond B Biol Sci 369, doi:10.1098/rstb.2013.0353 (2014).

Mayrose, I. et al. Methods for studying polyploid diversification and the dead end hypothesis: a reply to Soltis et al. (2014). New Phytol 206, 27-35, doi:10.1111/nph.13192 (2015).

Van de Peer, Y., Maere, S. \& Meyer, A. The evolutionary significance of ancient genome duplications. Nat Rev Genet 10, 725-732, doi:10.1038/nrg2600 (2009). 6ohaus, R. \& Van de Peer, Y. Of dups and dinos: 2791, doi:10.1105/tpc.114.126391 (2014).

Van de Peer, Y. Computational approaches to unveiling ancient genome duplications. Nat Rev Genet 5, 752-763, doi:10.1038/nrg1449 (2004).

Taylor, J. S. \& Raes, J. Duplication and divergence: the evolution of new genes and old ideas. Annu Rev Genet 38, 615-643, doi:10.1146/annurev.genet.38.072902.092831 (2004).

Bowers, J. E., Chapman, B. A., Rong, J. \& Paterson, A. H. Unravelling angiosperm genome evolution by phylogenetic analysis of chromosomal duplication events. Nature 422, 433-438, doi:10.1038/nature01521 (2003).

Conant, G. C. \& Wolfe, K. H. Turning a hobby into a job: how duplicated genes find new functions. Nat Rev Genet 9, 938-950, doi:10.1038/nrg2482 (2008).

Comai, L. The advantages and disadvantages of being polyploid. Nat Rev Genet 6, 836-846, doi:10.1038/nrg1711 (2005).

Soltis, D. E. et al. Polyploidy and angiosperm diversification. Am J Bot 96, 336-348, doi:10.3732/ajb.0800079 (2009). 
Madlung, A. Polyploidy and its effect on evolutionary success: old questions revisited with new tools. Heredity 110, 99-104 (2013).

28 Leitch, A. R. \& Leitch, I. J. Perspective - Genomic plasticity and the diversity of polyploid plants. Science 320, 481-483 (2008).

Bomblies, K. \& Madlung, A. Polyploidy in the Arabidopsis genus. Chromosome Res 22, 117-134, doi:10.1007/s10577-014-9416-x (2014).

Lavania, U. C. et al. Autopolyploidy differentially influences body size in plants, but facilitates enhanced accumulation of secondary metabolites, causing increased cytosine methylation. Plant J 71, 539-549, doi:10.1111/j.1365-313X.2012.05006.x (2012).

Shi, X., Zhang, C., Ko, D. K. \& Chen, Z. J. Genome-Wide Dosage-Dependent and -Independent Regulation Contributes to Gene Expression and Evolutionary Novelty in Plant Polyploids. Mol Biol Evol 32, 23512366, doi:10.1093/molbev/msv116 (2015).

Song, Q. \& Chen, Z. J. Epigenetic and developmental regulation in plant polyploids. Curr Opin Plant Biol 24, 101-109, doi:10.1016/j.pbi.2015.02.007 (2015).

Soltis, P. S., Liu, X., Marchant, D. B., Visger, C. J. \& Soltis, D. E. Polyploidy and novelty: Gottlieb's legacy. Philos Trans R Soc Lond B Biol Sci 369, doi:10.1098/rstb.2013.0351 (2014).

34 Parisod, C., Holderegger, R. \& Brochmann, C. Evolutionary consequences of autopolyploidy. New Phytol 186, 5-17, doi:10.1111/j.1469-8137.2009.03142.x (2010).

Zhang, H. et al. Transcriptome shock invokes disruption of parental expression-conserved genes in tetraploid wheat. Sci Rep 6, 26363, doi:10.1038/srep26363 (2016).

Doyle, J. J. et al. Evolutionary genetics of genome merger and doubling in plants. Annu Rev Genet 42 , 443-461, doi:10.1146/annurev.genet.42.110807.091524 (2008).

Schoenfelder, K. P. \& Fox, D. T. The expanding implications of polyploidy. J Cell Biol 209, 485-491, doi:10.1083/jcb.201502016 (2015). Nicotiana polyploids. Nat Plants 2, 16119, doi:10.1038/nplants.2016.119 (2016). doi:10.3732/ajb.1500529 (2016).

Gross, K. \& Schiestl, F. P. Are tetraploids more successful? Floral signals, reproductive success and floral isolation in mixed-ploidy populations of a terrestrial orchid. Ann Bot 115, 263-273, doi:10.1093/aob/mcu244 (2015).

McCarthy, E. W. et al. The effect of polyploidy and hybridization on the evolution of floral colour in Nicotiana (Solanaceae). Ann Bot 115, 1117-1131, doi:10.1093/aob/mcv048 (2015).

Edger, P. P. et al. The butterfly plant arms-race escalated by gene and genome duplications. Proc Natl Acad Sci U S A 112, 8362-8366, doi:10.1073/pnas.1503926112 (2015).

van den Bergh, E., Hofberger, J. A. \& Schranz, M. E. Flower power and the mustard bomb: Comparative analysis of gene and genome duplications in glucosinolate biosynthetic pathway evolution in Cleomaceae and Brassicaceae. Am J Bot 103, 1212-1222, doi:10.3732/ajb.1500445 (2016).

Vergara, F., Kikuchi, J. \& Breuer, C. Artificial Autopolyploidization Modifies the Tricarboxylic Acid Cycle and GABA Shunt in Arabidopsis thaliana Col-0. Sci Rep 6, 26515, doi:10.1038/srep26515 (2016). Oswald, B. P. \& Nuismer, S. L. Neopolyploidy and pathogen resistance. P Roy Soc B-Biol Sci 274, 23932397 (2007).

Hahn, M. A., van Kleunen, M. \& Muller-Scharer, H. Increased Phenotypic Plasticity to Climate May Have Boosted the Invasion Success of Polyploid Centaurea stoebe. Plos One 7 (2012).

Ramsey, J. Polyploidy and ecological adaptation in wild yarrow. Proc Natl Acad Sci U S A 108, 7096-7101, doi:10.1073/pnas.1016631108 (2011). to West African Acacia senegal (L.) Willd. Trees. Frontiers in Plant Science 7, doi:ARTN 821 10.3389/fpls.2016.00821 (2016). Chao, D. Y. et al. Polyploids exhibit higher potassium uptake and salinity tolerance in Arabidopsis. Science 341, 658-659, doi:10.1126/science.1240561 (2013). Ruiz, M. et al. Tetraploidy enhances the ability to exclude chloride from leaves in carrizo citrange seedlings. J Plant Physiol 205, 1-10, doi:10.1016/j.jplph.2016.08.002 (2016).

Yang, P. M., Huang, Q. C., Qin, G. Y., Zhao, S. P. \& Zhou, J. G. Different drought-stress responses in photosynthesis and reactive oxygen metabolism between autotetraploid and diploid rice. Photosynthetica 52, 193-202 (2014). 
Wood, T. E. et al. The frequency of polyploid speciation in vascular plants. Proc Natl Acad Sci U S A 106, 13875-13879, doi:10.1073/pnas.0811575106 (2009).

53 Barker, M. S., Arrigo, N., Baniaga, A. E., Li, Z. \& Levin, D. A. On the relative abundance of autopolyploids and allopolyploids. New Phytologist 210, 391-398 (2016). Fowler, N. L. \& Levin, D. A. Critical factors in the establishment of allopolyploids. Am J Bot, doi:10.3732/ajb.1500407 (2016).

Mayrose, I. et al. Recently formed polyploid plants diversify at lower rates. Science 333, 1257, doi:10.1126/science.1207205 (2011).

Oberlander, K. C., Dreyer, L. L., Goldblatt, P., Suda, J. \& Linder, H. P. Species-rich and polyploid-poor: Insights into the evolutionary role of whole-genome duplication from the Cape flora biodiversity hotspot. Am J Bot 103, 1336-1347, doi:10.3732/ajb.1500474 (2016).

Husband, B. C., Baldwin, S. J. \& Sabara, H. A. Direct vs. indirect effects of whole-genome duplication on prezygotic isolation in Chamerion angustifolium: Implications for rapid speciation. Am J Bot 103, 12591271, doi:10.3732/ajb.1600097 (2016).

Schranz, M. E., Mohammadin, S. \& Edger, P. P. Ancient whole genome duplications, novelty and diversification: the WGD Radiation Lag-Time Model. Curr Opin Plant Biol 15, 147-153, doi:10.1016/j.pbi.2012.03.011 (2012).

Tank, D. C. et al. Nested radiations and the pulse of angiosperm diversification: increased diversification rates often follow whole genome duplications. New Phytol 207, 454-467, doi:10.1111/nph.13491 (2015).

Clarke, J. T., Lloyd, G. T. \& Friedman, M. Little evidence for enhanced phenotypic evolution in early teleosts relative to their living fossil sister group. Proc Natl Acad Sci U S A, doi:10.1073/pnas.1607237113 (2016).

Robertson, F. M. et al. Lineage-specific rediploidization is a mechanism to explain time-lags between genome duplication and evolutionary diversification. BioRxiv submitted (2017). 544, doi:10.1098/rstb.2001.1035 (2002). Plant Biol 30, 25-32, doi:10.1016/j.pbi.2016.01.002 (2016).

Huang, C. H. et al. Multiple Polyploidization Events across Asteraceae with Two Nested Events in the Early History Revealed by Nuclear Phylogenomics. Mol Biol Evol, doi:10.1093/molbev/msw157 (2016). Barker, M. S. et al. Most Compositae (Asteraceae) are descendants of a paleohexaploid and all share a paleotetraploid ancestor with the Calyceraceae. Am J Bot 103, 1203-1211, doi:10.3732/ajb.1600113 (2016).

Crow, K. D., Wagner, G. P. \& Investigators, S. T.-N. Y. Proceedings of the SMBE Tri-National Young Investigators' Workshop 2005. What is the role of genome duplication in the evolution of complexity and diversity? Mol Biol Evol 23, 887-892, doi:10.1093/molbev/msj083 (2006).

Fawcett, J. A., Maere, S. \& Van de Peer, Y. Plants with double genomes might have had a better chance to survive the Cretaceous-Tertiary extinction event. Proc Natl Acad Sci U S A 106, 5737-5742, doi:10.1073/pnas.0900906106 (2009).

Vanneste, K., Baele, G., Maere, S. \& Van de Peer, Y. Analysis of 41 plant genomes supports a wave of successful genome duplications in association with the Cretaceous-Paleogene boundary. Genome Res 24, 1334-1347, doi:10.1101/gr.168997.113 (2014).

Cannon, S. B. et al. Multiple polyploidy events in the early radiation of nodulating and nonnodulating legumes. Mol Biol Evol 32, 193-210, doi:10.1093/molbev/msu296 (2015).

Renne, P. R. et al. State shift in Deccan volcanism at the Cretaceous-Paleogene boundary, possibly induced by impact. Science 350, 76-78, doi:10.1126/science.aac7549 (2015). Petersen, S. V., Dutton, A. \& Lohmann, K. C. End-Cretaceous extinction in Antarctica linked to both Deccan volcanism and meteorite impact via climate change. Nat Commun 7, 12079, doi:10.1038/ncomms12079 (2016).

Li, Z. et al. Early genome duplications in conifers and other seed plants. Sci Adv 1, e1501084, doi:10.1126/sciadv.1501084 (2015).

Wille, M., Nagler, T. F., Lehmann, B., Schroder, S. \& Kramers, J. D. Hydrogen sulphide release to surface waters at the Precambrian/Cambrian boundary. Nature 453, 767-769, doi:10.1038/nature07072 (2008). Hurley, I. A. et al. A new time-scale for ray-finned fish evolution. Proc Biol Sci 274, 489-498 (2007).

Soltis, D. E. \& Burleigh, J. G. Surviving the K-T mass extinction: new perspectives of polyploidization in angiosperms. Proc Natl Acad Sci U S A 106, 5455-5456, doi:10.1073/pnas.0901994106 (2009). 

Sci U S A 111, 15149-15154, doi:10.1073/pnas.1404177111 (2014).

77 Edwards, E. J. et al. The origins of C4 grasslands: integrating evolutionary and ecosystem science. Science 328, 587-591, doi:10.1126/science.1177216 (2010).

78 Stebbins, G. L. Polyploidy and the Distribution of the Arctic-Alpine Flora - New Evidence and a New Approach. Bot Helv 94, 1-13 (1984). niches characterize polyploid species of European primroses in Primula sect. Aleuritia. J Biogeogr 40, 1278-1289, doi:10.1111/jbi.12085 (2013).

Hovick, S. M. \& Whitney, K. D. Hybridisation is associated with increased fecundity and size in invasive taxa: meta-analytic support for the hybridisation-invasion hypothesis. Ecol Lett 17, 1464-1477, doi:10.1111/ele.12355 (2014).

Bock, D. G. et al. What we still don't know about invasion genetics. Mol Ecol 24, 2277-2297, doi:10.1111/mec.13032 (2015).

Pandit, M. K., Pocock, M. J. O. \& Kunin, W. E. Ploidy influences rarity and invasiveness in plants. J Ecol 99, 1108-1115 (2011).

Parisod, C. \& Broennimann, O. Towards unified hypotheses of the impact of polyploidy on ecological niches. New Phytol 212, 540-542, doi:10.1111/nph.14133 (2016).

Glennon, K. L., Ritchie, M. E. \& Segraves, K. A. Evidence for shared broad-scale climatic niches of diploid and polyploid plants. Ecol Lett 17, 574-582 (2014).

Marchant, B. D., Soltis, D. E. \& Soltis, P. S. Patterns of abiotic niche shifts in allopolyploids relative to their progenitors. New Phytol, doi:10.1111/nph.14069 (2016).

Jiao, Y. et al. Ancestral polyplo

Vekemans, D. et al. Gamma paleohexaploidy in the stem lineage of core eudicots: significance for MADSbox gene and species diversification. Mol Biol Evol 29, 3793-3806, doi:10.1093/molbev/mss183 (2012). Ming, R. et al. The pineapple genome and the evolution of CAM photosynthesis. Nat Genet 47, 14351442, doi:10.1038/ng.3435 (2015).

Soltis, P. S. \& Soltis, D. E. Ancient WGD events as drivers of key innovations in angiosperms. Curr Opin Plant Biol 30, 159-165, doi:10.1016/j.pbi.2016.03.015 (2016).

Nakatani, Y., Takeda, H., Kohara, Y. \& Morishita, S. Reconstruction of the vertebrate ancestral genome reveals dynamic genome reorganization in early vertebrates. Genome Res 17, 1254-1265, doi:10.1101/gr.6316407 (2007).

Makino, T. \& Mclysaght, A. Ohnologs in the human genome are dosage balanced and frequently associated with disease. Proc Natl Acad Sci U S A 107, 9270-9274, doi:10.1073/pnas.0914697107 (2010). Singh, P. P. et al. On the expansion of "dangerous" gene repertoires by whole-genome duplications in early vertebrates. Cell Rep 2, 1387-1398, doi:10.1016/j.celrep.2012.09.034 (2012). Duplication in Early Vertebrates, Based on Synteny Comparison across Multiple Genomes. PLoS Comput Biol 11, e1004394, doi:10.1371/journal.pcbi.1004394 (2015).

Berthelot, C. et al. The rainbow trout genome provides novel insights into evolution after whole-genome duplication in vertebrates. Nat Commun 5, 3657, doi:10.1038/ncomms4657 (2014). Session, A. M. et al. Genome evolution in the allotetraploid frog Xenopus laevis. Nature 538, 336-343, doi:10.1038/nature19840 (2016).

96 Li, Z. et al. Gene Duplicability of Core Genes Is Highly Consistent across All Angiosperms. Plant Cell 28, 326-344, doi:10.1105/tpc.15.00877 (2016).

97 Maere, S. et al. Modeling gene and genome duplications in eukaryotes. Proc Natl Acad Sci U S A 102, 5454-5459, doi:10.1073/pnas.0501102102 (2005).

Freeling, M. Bias in plant gene content following different sorts of duplication: tandem, whole-genome, segmental, or by transposition. Annu Rev Plant Biol 60, 433-453, doi:10.1146/annurev.arplant.043008.092122 (2009).

99 Conant, G. C. Comparative genomics as a time machine: how relative gene dosage and metabolic requirements shaped the time-dependent resolution of yeast polyploidy. Mol Biol Evol 31, 3184-3193, doi:10.1093/molbev/msu250 (2014).

100 Birchler, J. A. \& Veitia, R. A. Gene balance hypothesis: connecting issues of dosage sensitivity across biological disciplines. Proc Natl Acad Sci U S A 109, 14746-14753, doi:10.1073/pnas.1207726109 (2012). 

duplication events. Curr Opin Plant Biol 15, 168-176, doi:10.1016/j.pbi.2012.01.003 (2012).

102 Zhou, W. et al. Evolution of herbivore-induced early defense signaling was shaped by genome-wide duplications in Nicotiana. Elife 5, doi:10.7554/eLife.19531 (2016).

103 Schmitz, J. F., Zimmer, F. \& Bornberg-Bauer, E. Mechanisms of transcription factor evolution in Metazoa. Nucleic Acids Res 44, 6287-6297, doi:10.1093/nar/gkw492 (2016).

104 Edgar, B. A., Zielke, N. \& Gutierrez, C. Endocycles: a recurrent evolutionary innovation for post-mitotic cell growth. Nat Rev Mol Cell Biol 15, 197-210, doi:10.1038/nrm3756 (2014).

105 De Veylder, L., Larkin, J. C. \& Schnittger, A. Molecular control and function of endoreplication in development and physiology. Trends Plant Sci 16, 624-634, doi:10.1016/j.tplants.2011.07.001 (2011).

106 Orr-Weaver, T. L. When bigger is better: the role of polyploidy in organogenesis. Trends Genet 31, 307315, doi:10.1016/j.tig.2015.03.011 (2015).

107 Cho, E. H. \& Nijhout, H. F. Development of polyploidy of scale-building cells in the wings of Manduca sexta. Arthropod Struct Dev 42, 37-46, doi:10.1016/j.asd.2012.09.003 (2013).

108 Davoli, T. \& de Lange, T. The causes and consequences of polyploidy in normal development and cancer. Annu Rev Cell Dev Biol 27, 585-610, doi:10.1146/annurev-cellbio-092910-154234 (2011).

109 Ceccarelli, M., Santantonio, E., Marmottini, F., Amzallag, G. N. \& Cionini, P. G. Chromosome endoreduplication as a factor of salt adaptation in Sorghum bicolor. Protoplasma 227, 113-118, doi:10.1007/s00709-005-0144-0 (2006).

110 Yant, L. \& Bomblies, K. Genome management and mismanagement--cell-level opportunities and challenges of whole-genome duplication. Genes Dev 29, 2405-2419, doi:10.1101/gad.271072.115 (2015).

111 Coward, J. \& Harding, A. Size Does Matter: Why Polyploid Tumor Cells are Critical Drug Targets in the War on Cancer. Front Oncol 4, 123, doi:10.3389/fonc.2014.00123 (2014).

112 Fujiwara, T. et al. Cytokinesis failure generating tetraploids promotes tumorigenesis in p53-null cells. Nature 437, 1043-1047, doi:10.1038/nature04217 (2005).

113 LV, L. et al. Tetraploid cells from cytokinesis failure induce aneuploidy and spontaneous transformation of mouse ovarian surface epithelial cells. Cell Cycle 11, 2864-2875, doi:10.4161/cc.21196 (2012).

114 Caldwell, C. M., Green, R. A. \& Kaplan, K. B. APC mutations lead to cytokinetic failures in vitro and tetraploid genotypes in Min mice. J Cell Biol 178, 1109-1120, doi:10.1083/jcb.200703186 (2007).

115 Dikovskaya, D. et al. Loss of APC induces polyploidy as a result of a combination of defects in mitosis and apoptosis. J Cell Biol 176, 183-195, doi:10.1083/jcb.200610099 (2007).

116 Borodkina, A. V. et al. Tetraploidization or autophagy: The ultimate fate of senescent human endometrial stem cells under ATM or p53 inhibition. Cell Cycle 15, 117-127, doi:10.1080/15384101.2015.1121326 (2016).

117 Kuznetsova, A. Y. et al. Chromosomal instability, tolerance of mitotic errors and multidrug resistance are promoted by tetraploidization in human cells. Cell Cycle 14, 2810-2820, doi:10.1080/15384101.2015.1068482 (2015).

118 Mardin, B. R. et al. A cell-based model system links chromothripsis with hyperploidy. Mol Syst Biol 11, 828, doi:10.15252/msb.20156505 (2015).

119 de Bruin, E. C. et al. Spatial and temporal diversity in genomic instability processes defines lung cancer evolution. Science 346, 251-256, doi:10.1126/science.1253462 (2014).

120 Rode, A., Maass, K. K., Willmund, K. V., Lichter, P. \& Ernst, A. Chromothripsis in cancer cells: An update. Int J Cancer 138, 2322-2333, doi:10.1002/ijc.29888 (2016).

121 Gerstein, A. C. et al. Polyploid titan cells produce haploid and aneuploid progeny to promote stress adaptation. MBio 6, e01340-01315, doi:10.1128/mBio.01340-15 (2015).

122 Yona, A. H. et al. Chromosomal duplication is a transient evolutionary solution to stress. Proc Natl Acad Sci U S A 109, 21010-21015, doi:10.1073/pnas.1211150109 (2012).

123 Gordon, D. J., Resio, B. \& Pellman, D. Causes and consequences of aneuploidy in cancer. Nat Rev Genet 13, 189-203, doi:10.1038/nrg3123 (2012).

124 Kaya, A. et al. Adaptive aneuploidy protects against thiol peroxidase deficiency by increasing respiration via key mitochondrial proteins. Proc Natl Acad Sci U S A 112, 10685-10690, doi:10.1073/pnas.1505315112 (2015).

125 Selmecki, A. M. et al. Polyploidy can drive rapid adaptation in yeast. Nature 519, 349-352, doi:10.1038/nature14187 (2015).

126 Voordeckers, K. et al. Adaptation to High Ethanol Reveals Complex Evolutionary Pathways. PLoS Genet 11, e1005635, doi:10.1371/journal.pgen.1005635 (2015). 
Tanaka, K. \& Hirota, T. Chromosomal instability: A common feature and a therapeutic target of cancer. Biochim Biophys Acta 1866, 64-75, doi:10.1016/j.bbcan.2016.06.002 (2016).

128 Santaguida, S. \& Amon, A. Short- and long-term effects of chromosome mis-segregation and aneuploidy. Nat Rev Mol Cell Biol 16, 473-485, doi:10.1038/nrm4025 (2015).

129 Dewhurst, S. M. et al. Tolerance of whole-genome doubling propagates chromosomal instability and accelerates cancer genome evolution. Cancer Discov 4, 175-185, doi:10.1158/2159-8290.CD-13-0285 (2014).

130 Barrick, J. E. \& Lenski, R. E. Genome dynamics during experimental evolution. Nat Rev Genet 14, 827839, doi:10.1038/nrg3564 (2013).

131 Breuert, S., Allers, T., Spohn, G. \& Soppa, J. Regulated polyploidy in halophilic archaea. PLoS One 1, e92, doi:10.1371/journal.pone.0000092 (2006).

132 Pecoraro, V., Zerulla, K., Lange, C. \& Soppa, J. Quantification of ploidy in proteobacteria revealed the existence of monoploid, (mero-)oligoploid and polyploid species. PLoS One 6, e16392, doi:10.1371/journal.pone.0016392 (2011).

133 Soppa, J. Polyploidy and community structure. Nat Microbiol 2, 16261, doi:10.1038/nmicrobiol.2016.261 (2017).

134 Soppa, J. Ploidy and gene conversion in Archaea. Biochem Soc Trans 39, 150-154, doi:10.1042/BST0390150 (2011).

135 Lange, C., Zerulla, K., Breuert, S. \& Soppa, J. Gene conversion results in the equalization of genome copies in the polyploid haloarchaeon Haloferax volcanii. Mol Microbiol 80, 666-677, doi:10.1111/j.13652958.2011.07600.x (2011).

136 Maciver, S. K. Asexual Amoebae Escape Muller's Ratchet through Polyploidy. Trends Parasitol 32, 855862, doi:10.1016/j.pt.2016.08.006 (2016).

137 Markov, A. V. \& Kaznacheev, I. S. Evolutionary consequences of polyploidy in prokaryotes and the origin of mitosis and meiosis. Biol Direct 11, 28, doi:10.1186/s13062-016-0131-8 (2016).

138 Ainouche, M. L. \& Jenczewski, E. Focus on polyploidy. New Phytologist 186, 1-4, doi:DOI 10.1111/j.14698137.2010.03215.x (2010).

139 Barker, M. S., Husband, B. C. \& Pires, J. C. Spreading Winge and flying high: the evolutionary importance of polyploidy after a century of study. Am J Bot 103, 1139-1145 (2016).

140 Mable, B. K. Polyploids and hybrids in changing environments: winners or losers in the struggle for adaptation? Heredity 110, 95-96, doi:10.1038/hdy.2012.105 (2013).

141 Soltis, D. E., Visger, C. J., Marchant, D. B. \& Soltis, P. S. Polyploidy: Pitfalls and paths to a paradigm. Am J Bot, doi:10.3732/ajb.1500501 (2016).

142 Soltis, D. E., Buggs, R. J. A., Doyle, J. J. \& Soltis, P. S. What we still don't know about polyploidy. Taxon 59, 1387-1403 (2010).

143 Poustka, A. J. et al. A global view of gene expression in lithium and zinc treated sea urchin embryos: new components of gene regulatory networks. Genome Bio/ 8, R85, doi:10.1186/gb-2007-8-5-r85 (2007).

144 Ramsey, J. \& Schemske, D. W. Pathways, mechanisms, and rates of polyploid formation in flowering plants. Annu Rev Ecol Syst 29, 467-501, doi:DOI 10.1146/annurev.ecolsys.29.1.467 (1998).

145 Hu, G., Koh, J., Yoo, M. J., Chen, S. \& Wendel, J. F. Gene-expression novelty in allopolyploid cotton: a proteomic perspective. Genetics 200, 91-104, doi:10.1534/genetics.115.174367 (2015).

146 Yoo, M. J., Liu, X., Pires, J. C., Soltis, P. S. \& Soltis, D. E. Nonadditive gene expression in polyploids. Annu Rev Genet 48, 485-517, doi:10.1146/annurev-genet-120213-092159 (2014).

147 Gallagher, J. P., Grover, C. E., Hu, G. \& Wendel, J. F. Insights into the Ecology and Evolution of Polyploid Plants through Network Analysis. Mol Ecol 25, 2644-2660, doi:10.1111/mec.13626 (2016).

148 Kreiner, J. M., Kron, P. \& Husband, B. C. Frequency and maintenance of unreduced gametes in natural plant populations: associations with reproductive mode, life history and genome size. New Phytol, doi:10.1111/nph.14423 (2017).

149 Pecrix, Y. et al. Polyploidization mechanisms: temperature environment can induce diploid gamete formation in Rosa sp. J Exp Bot 62, 3587-3597, doi:10.1093/jxb/err052 (2011).

150 De Storme, N., Copenhaver, G. P. \& Geelen, D. Production of diploid male gametes in Arabidopsis by cold-induced destabilization of postmeiotic radial microtubule arrays. Plant Physiol 160, 1808-1826, doi:10.1104/pp.112.208611 (2012).

151 Mason, A. S., Nelson, M. N., Yan, G. \& Cowling, W. A. Production of viable male unreduced gametes in Brassica interspecific hybrids is genotype specific and stimulated by cold temperatures. BMC Plant Biol 11, 103, doi:10.1186/1471-2229-11-103 (2011). 
152 Kurschner, W. M., Batenburg, S. J. \& Mander, L. Aberrant Classopollis pollen reveals evidence for unreduced (2n) pollen in the conifer family Cheirolepidiaceae during the Triassic-Jurassic transition. Proc Biol Sci 280, 20131708, doi:10.1098/rspb.2013.1708 (2013).

153 Foster, C. B. \& Afonin, S. A. Abnormal pollen grains: an outcome of deteriorating atmospheric conditions around the Permian - Triassic boundary. J Geol Soc London 162, 653-659, doi:Doi 10.1144/0016-764904047 (2005).

154 Visscher, H. et al. Environmental mutagenesis during the end-Permian ecological crisis. P Natl Acad Sci USA 101, 12952-12956, doi:10.1073/pnas.0404472101 (2004).

155 Freeling, M. The distribution of ancient polyploidies in the plant phylogenetic tree is a spandrel of occasional sex. Plant Cell (in press) (2017).

156 Pierazzo, E. et al. Ozone perturbation from medium-size asteroid impacts in the ocean. Earth Planet Sc Lett 299, 263-272, doi:10.1016/j.epsl.2010.08.036 (2010).

157 Ruhfel, B. R., Gitzendanner, M. A., Soltis, P. S., Soltis, D. E. \& Burleigh, J. G. From algae to angiospermsinferring the phylogeny of green plants (Viridiplantae) from 360 plastid genomes. BMC Evol Biol 14, 23, doi:10.1186/1471-2148-14-23 (2014).

158 Magallon, S. A Review of the Effect of Relaxed Clock Method, Long Branches, Genes, and Calibrations in the Estimation of Angiosperm Age. Bot Sci 92, 1-22 (2014).

159 Cai, J. et al. The genome sequence of the orchid Phalaenopsis equestris. Nat Genet 47, 65-72, doi:10.1038/ng.3149 (2015).

160 Olsen, J. L. et al. The genome of the seagrass Zostera marina reveals angiosperm adaptation to the sea. Nature 530, 331-335, doi:10.1038/nature16548 (2016).

161 Myburg, A. A. et al. The genome of Eucalyptus grandis. Nature 510, 356-362, doi:10.1038/nature13308 (2014).

162 Vanneste, K., Sterck, L., Myburg, A. A., Van de Peer, Y. \& Mizrachi, E. Horsetails Are Ancient Polyploids: Evidence from Equisetum giganteum. Plant Cell 27, 1567-1578, doi:10.1105/tpc.15.00157 (2015).

163 Kenny, N. J. et al. Ancestral whole-genome duplication in the marine chelicerate horseshoe crabs. Heredity (Edinb) 116, 190-199, doi:10.1038/hdy.2015.89 (2016).

164 Lien, S. et al. The Atlantic salmon genome provides insights into rediploidization. Nature 533, 200-205, doi:10.1038/nature17164 (2016).

165 Iorizzo, M. et al. A high-quality carrot genome assembly provides new insights into carotenoid accumulation and asterid genome evolution. Nat Genet 48, 657-666, doi:10.1038/ng.3565 (2016).

166 Corrochano, L. M. et al. Expansion of Signal Transduction Pathways in Fungi by Extensive Genome Duplication. Curr Biol 26, 1577-1584, doi:10.1016/j.cub.2016.04.038 (2016).

167 Bredeson, J. V. et al. Sequencing wild and cultivated cassava and related species reveals extensive interspecific hybridization and genetic diversity. Nat Biotechnol 34, 562-570, doi:10.1038/nbt.3535 (2016).

168 Hoshino, A. et al. Genome sequence and analysis of the Japanese morning glory Ipomoea nil. Nat Commun 7, 13295, doi:10.1038/ncomms13295 (2016).

169 Sollars, E. S. et al. Genome sequence and genetic diversity of European ash trees. Nature 541, 212-216, doi:10.1038/nature20786 (2017).

170 Van de Peer, Y., Fawcett, J. A., Proost, S., Sterck, L. \& Vandepoele, K. The flowering world: a tale of duplications. Trends Plant Sci 14, 680-688, doi:10.1016/j.tplants.2009.09.001 (2009).

171 Hijmans, R. J., Cameron, S. E., Parra, J. L., Jones, P. G. \& Jarvis, A. Very high resolution interpolated climate surfaces for global land areas. Int J Climatol 25, 1965-1978 (2005).

172 Yant, L. et al. Meiotic adaptation to genome duplication in Arabidopsis arenosa. Curr Biol 23, 2151-2156, doi:10.1016/j.cub.2013.08.059 (2013).

\section{Acknowledgements}

YVdP and KM acknowledge the Multidisciplinary Research Partnership "Bioinformatics: from nucleotides to networks" Project (no. 01MR0310W) of Ghent University. YVdP acknowledges funding from the European Union Seventh Framework Programme (FP7/2007-2013) under European Research Council Advanced Grant Agreement 322739 - DOUBLEUP. KM acknowledges the Fonds voor Wetenschappelijk Onderzoek - Flanders (FWO15/PRJ/396). Special thanks go to Rolf Lohaus for helpful 
discussions and to Polina Novikova for providing Figure 3. YVdP, EM, and KM also thank the University of Pretoria for general support. All authors want to apologize to the many whose work was overlooked or could not be included due to space constraints. Finally, the authors want to thank four anonymous reviewers for their comments and suggestions that greatly helped improving the paper.

\section{BOX 1. Allopolyploidy versus autopolyploidy}

Polyploids are traditionally classified as either autopolyploids, arisen within a single species by doubling of one chromosome set, or allopolyploids, arisen through the merging - and subsequent doubling - of structurally different chromosome sets. Several different scenarios for the formation of both autopolyploids and allopolyploids have been proposed ${ }^{144}$. Allopolyploidy has long been associated with changes in gene expression and epigenetic remodelling ${ }^{145,146}$, and has generally been expected to result in higher adaptive potential than autopolyploidy. Under a hybridization scenario, homologs originate from species that have already diverged and thus sequence and functional differences are expected from the start, increasing genetic variation and potentially providing the polyploid with an initial selective advantage. Novel allelic combinations brought about by the merging of two different species might be responsible for hybrid vigour or heterosis leading to the formation of more extreme phenotypes in the hybrid population ${ }^{24}$. On the other hand, genome duplication, as in autopolyploids, can also lead to immediate changes in gene expression, physiology and ecological tolerance ${ }^{29,34,147}$. So, although the (potentially different) effects of both allopolyploidy and autopolyploidy have been discussed at large, which and how many genes change their expression, and which and how many are silenced, remains unclear, and often seems species-dependent.

Most polyploids arise through the formation of unreduced gametes 2,29,144,148. Interestingly, the levels of unreduced gametes can be increased by external stimuli. For instance, increasing temperatures in roses increased the production of unreduced gametes ${ }^{149}$. Similarly, the induction of cold stress increased the formation of unreduced gametes in Arabidopsis ${ }^{150}$ and in Brassica interspecific hybrids ${ }^{151}$. Also in amphibians and fishes, polyploidy - through the production of unreduced gametes - can be induced by temperature shock ${ }^{2}$. Interestingly, increased levels of fossil unreduced pollen were also observed in a now extinct conifer family at the Triassic-Jurassic transition, which corresponds to the fourth of the five major extinction events ${ }^{152}$. In addition, atypical gymnosperm pollen ${ }^{153}$ and lycophyte spores ${ }^{154}$ have been found at the Permian-Triassic boundary, formed by the third of the five major extinction events. In line with this, the increased formation of unreduced gametes could thus also have been an important factor in the apparent clustering of palaeopolyploidizations at the K-Pg boundary, the fifth and most recent of the last five major mass extinctions ${ }^{68}$.

\section{BOX 2. Polyploidy, a spandrel of occasional sex}

Evidence is accumulating that polyploidy can increase mutational and environmental robustness, which might increase the potential for specific adaptation under changing environmental conditions or reduce the risk of extinction during periods of environmental upheaval. Recently, Freeling ${ }^{155}$ proposed an interesting alternative hypothesis about how or why polyploids might be able to survive periods of mass extinction (Fig. 1, K/Pg boundary). Freeling started from the observation that new polyploids encounter major difficulties during sexual reproduction due to meiotic difficulties ${ }^{24}$. This reduces fitness if organisms reproduce sexually. Furthermore, an asteroid impacting Earth would cause depletion of the Earth's protective ozone layer ${ }^{156}$, which in turn leads to a serious increase in surface UV-B irradiance causing mutation and breakage of DNA. If the polyploid could however shield its reproductive meristems and avoid meiosis altogether, and reproduce asexually for a while, it would increase its chances of 
survival ${ }^{155}$. During, or after its 'hiding out' under unfavourable conditions within the soma of plants that reproduce asexually, for instance by budding underground or under water, the polyploid can then undergo diploidization, which is necessary for again establishing accurate meiosis. When meiosis has been re-established, the 'rediploidized' polyploid (now diploid) might then again produce flowers and reproduce sexually. Freeling thus proposes that the observed pattern of clustered WGDs at times of mass extinctions can be explained by considering polyploidy as a mere spandrel, a by-product of an asexual life stage. Once the environment has normalized, the now diploidized polyploid does not need to be asexual anymore, but can reproduce sexually, necessary for its long term evolutionary success. Of course, also non-polyploid asexuals should be able to survive mass extinctions by hiding out temporarily, but are expected to be outcompeted by the polyploids, because of the greater adaptive potential and increased colonization success of the latter ( ${ }^{155}$ and main text). 
Figure 1. Phylogenetic tree with known WGDs mapped. Pruned tree for plants ${ }^{157,158}$, animals, and fungi showing the evolutionary relationship between species for which the genome sequence - or extensive transcriptome data - are available and which are representative for the topic of polyploidy discussed in the current paper. WGDs described in previous studies ${ }^{68,72,88,159-169}$ are mapped onto the tree (red rectangles) to the best of our knowledge. WGDs estimated between 55 and 75 million years old (shaded area around the $\mathrm{K} / \mathrm{Pg}$ boundary) are indicated by pink rectangles. See text for details.

Fig. 2. Evolution of fitness and number of genes without and with WGD under times of environmental stress. Although the number of genes might continuously increase with time because of small-scale gene duplication ${ }^{97,170}$ - fluctuations might occur because of stress and selection for genes important for adaptation (panel top left) -, WGD will initially double the total number of genes (top right). Although gene loss following WGDs is usually extensive ${ }^{97}$, the biased retention of genes creating a regulatory and developmental spandrel ${ }^{98}$ might be responsible for long-term increase in biological complexity (see long term effects of WGDs). When the level of environmental stress (drastically) increases, we can expect fitness to decrease (bottom left). However, following WGD, polyploids might have increased adaptive potential, for instance due to increased genetic variation and changes in gene expression, preventing large drops in fitness (bottom right).

Figure 3. Distribution of diploid and tetraploid Neobatrachus species. The genus Neobatrachus consists of 10 extant species of Australian frogs: six diploid (N. fulvus, N. pelobatoides, N. pictus, N. sutor, N. wilsmorei, N. albipes; $2 \mathrm{n}=24$ ) and four tetraploid (N. aquilonius, N. centralis, N. kunapalari, N. sudelli; $4 n=48$ ). (A, B) Occurrence data (AmphibiaWeb: Information on amphibian biology and conservation. Berkeley, California: http://amphibiaweb.org/) of Neobatrachus species combined with climate data ${ }^{171}$ shows ecological differences between diploid and tetraploid species, the latter occupying areas with lower annual precipitation (** Mann-Whitney test $p$-value $<0.01$ ). This suggests that tetraploids are better adapted to drier regions and could escape to (invade) new and harsher ecological niches.

Fig. 4. Polyploidy in different systems. Left panel: both neutral and adaptive processes can contribute to the formation and establishment of polyploids. Environmental change or stress can increase the formation of unreduced gametes, while other processes such as hybridization can also contribute to an overall increase of unreduced gametes in the total gamete pool. However, for polyploids to survive and get established, selection for specific traits resulting from increased phenotypic variability and plasticity is probably still necessary. Also specific adaptation of more crucial cellular processes, such as meiosis, might be necessary for polyploids to become successful on the somewhat longer term ${ }^{172}$. Note that, in both the neutral and adaptive scenario, the environment plays an important role in polyploid establishment. Middle panel: shows the adaptive processes following a WGD in eukaryotic clonal systems such as cancer. WGD usually originates through mitotic errors, sometimes as a result of driver mutations, resulting in a tetraploid state. Further mutations in, for example, DNA damage response (e.g. p53) enable polyploid cells to further proliferate. However, cell divisions are unstable (chromosomal instability (CIN)) and give rise to aneuploidy. Since aneuploidies often result in lethal phenotypes, mutational robustness provided by the polyploid background allows near tetraploid cells to better tolerate aneuploidy. Such aneuploidy increases the genetic variation that can then result in the origin of tumorigenesis and further adaptive events (drug resistance, metastasis, etc....).Right panel: shows 
some of the hypothetical adaptive effects of polyploidy in prokaryotes (and possibly asexual eukaryotes ${ }^{136}$ ). Many prokaryotes display polyploidy where polyploidy levels are growth phase dependent. In prokaryotes, polyploidy can contribute to the direct survival of the individual (by serving as a phosphate reserve, by protecting against highly mutational environments, etc. ...) but can also contribute to the long term survival under varying selection regimes by increasing the genetic variation. Gene conversion would play an important role in establishing the adaptive benefits of polyploidy in prokaryotes.

\section{Glossary}

Assortative mating: Mating pattern in which individuals with common traits prefer to mate with one another. Here, polyploids mating with other polyploids, rather than with diploids, which might be incompatible.

Neofunctionalization: the process by which a gene acquires a novel gene function after a duplication event.

Sympatric speciation: the process through which new species evolve from a single ancestral species while occupying the same habitat

Ecological tolerance: the range of conditions - or niche breadth - that an organism can thrive in. More tolerant organisms can withstand a broader range of environmental conditions.

Key innovation: an important adaptation that leads to subsequent species radiation or is of major importance for the success of a taxonomic group.

Heterosis: The tendency of a hybrid to show qualities superior to those of its parents.

Chromothripsis: The phenomenon by which potentially thousands of chromosomal rearrangements occur in a single event in localised and confined regions in the genome. 

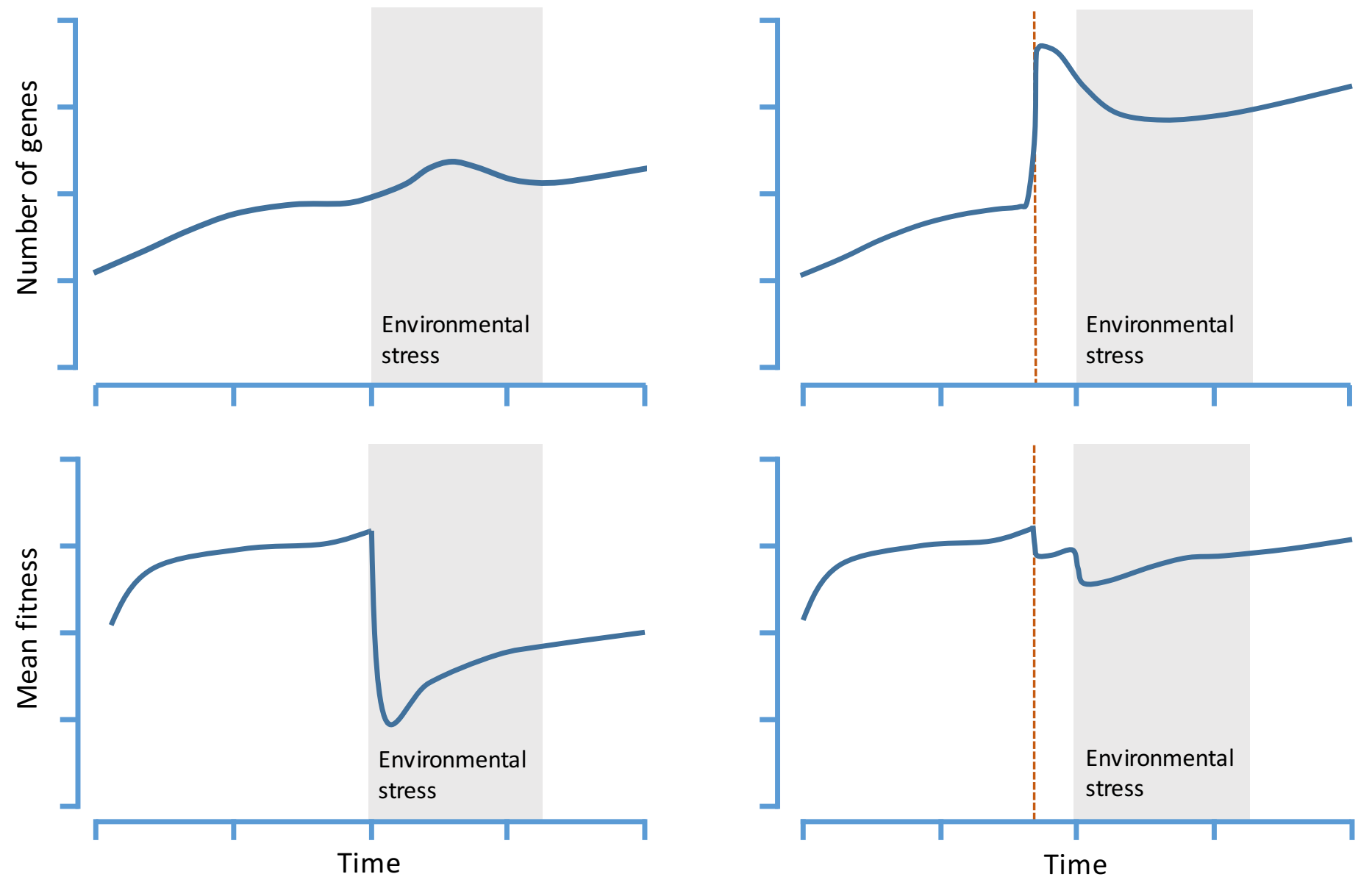
A
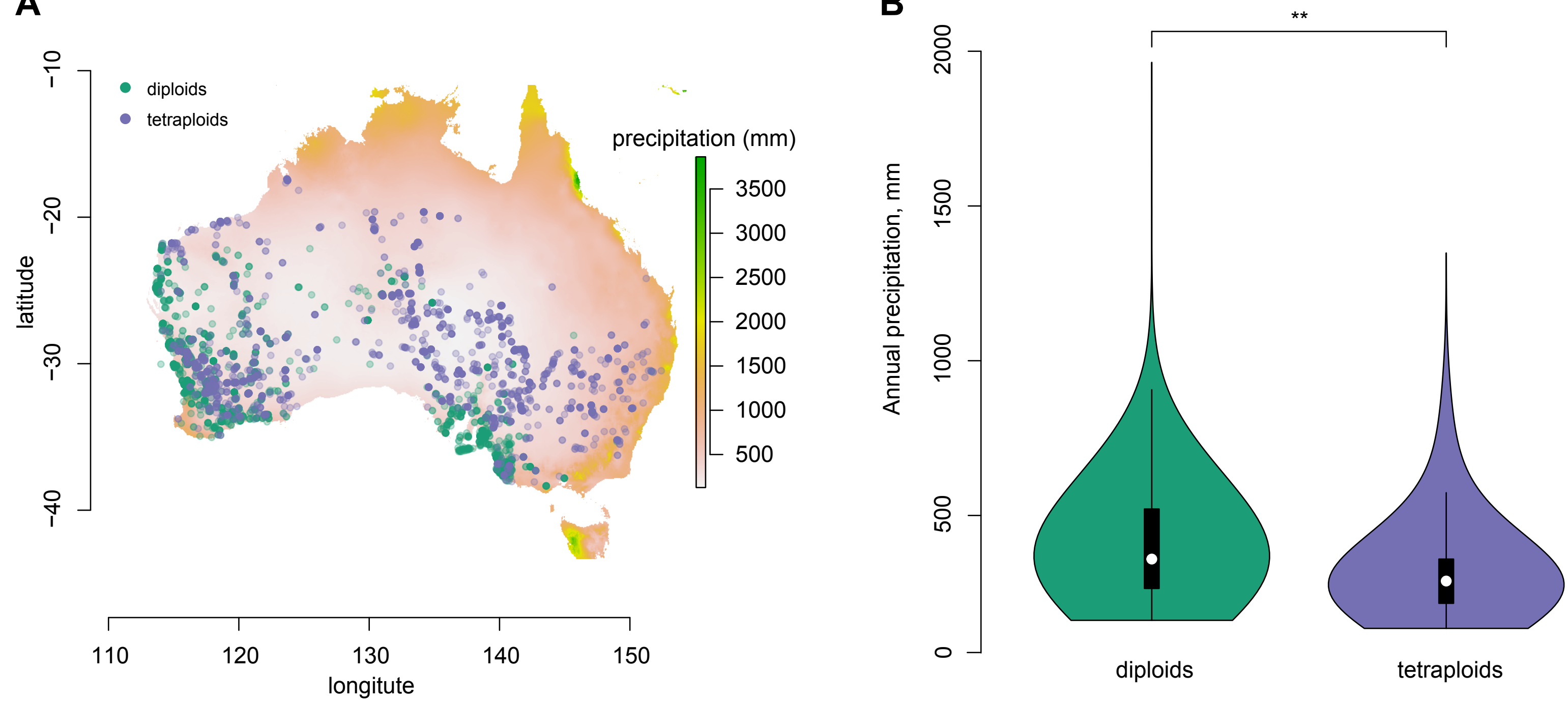

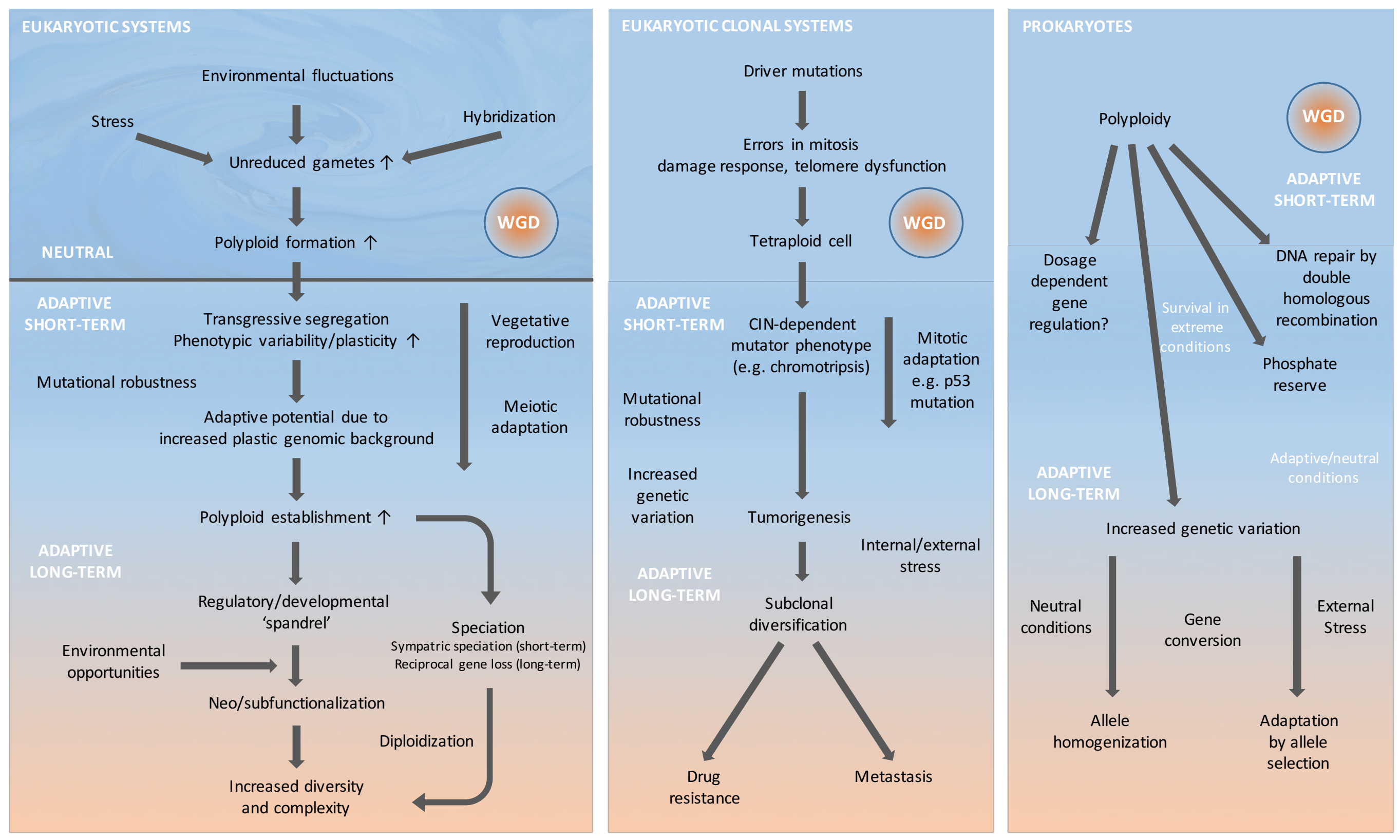